NUC-MINN-96/4-T

\title{
Redundance of $\Delta$-isobar Parameters in Effective Field Theories
}

\author{
Hua-Bin Tang and Paul J. Ellis \\ School of Physics and Astronomy, University of Minnesota, Minneapolis, MN 55455.
}

(March 14, 1996)

\begin{abstract}
It is shown that the off-shell parameters in the interaction Lagrangian of pions, nucleons, and $\Delta$-isobars are redundant in the framework of effective field theories. Our results also suggest the necessity of including the $\Delta$ as an explicit dynamical degree of freedom.
\end{abstract}

The spin- $\frac{3}{2}$ isospin- $\frac{3}{2} \Delta$ isobar plays an important role in fitting pion-nucleon and nucleonnucleon scattering data. However, a satisfactory covariant treatment of spin- $\frac{3}{2}$ fields has been hampered [1] by the existence of two parameters commonly called $A$ and $Z$. Nath et al. [2] have argued that physical quantities are independent of $A$, while they depend on the off-shell parameter $Z$ which occurs in the $\pi N \Delta$ interaction. They further suggested that quantum theory requires $Z=\frac{1}{2}$. Olsson and Osypowski [3] mentioned another claim that both $Z= \pm \frac{1}{2}$ are possible. Various choices of the $Z$ parameter, including fitting it to experimental data, have been discussed in Ref. [4]. In a recent fit [0] to $\pi N$ scattering data, a value of $Z \sim-\frac{1}{2}$ was favored.

Notice that the above models were not in the framework of modern effective field theories (EFTs) [6], of which chiral perturbation theory (ChPT) [7] is a successful prototype. Nevertheless, the role of the off-shell parameters in modern EFTs is also of interest. Recently, Banerjee and Milana [8] have examined $1 / M$ corrections (where $M$ is a typical baryon mass) in heavy-baryon chiral perturbation theory (ChPT) [9]. They suggested, on the one hand, 
that one may be at an impasse without knowledge of the off-shell parameters and, on the other hand, that the "off-shell" $\Delta$ effects could be absorbed as part of the higher-dimension operators using purely "on-shell" baryon fields. In this Letter, we show that the off-shell parameter in the $\pi N \Delta$ interaction, and similar parameters which arise for the $\pi \Delta \Delta$ interaction, can be subsumed in other terms in the effective Lagrangian. We demonstate further that the $\Delta$ isobar should be treated as a dynamical degree of freedom in agreement with the view taken in e.g. Refs. [8, 10,11], but at variance with that of Bernard et al. [12].

The free Lagrangian for the spin- $\frac{3}{2}$ and isospin- $\frac{3}{2}$ vector-spinor field

$$
\Delta_{\mu}=\left(\begin{array}{c}
\Delta_{\mu}^{++} \\
\Delta_{\mu}^{+} \\
\Delta_{\mu}^{0} \\
\Delta_{\mu}^{-}
\end{array}\right)
$$

is given by [13]

$$
\mathcal{L}_{0}=\bar{\Delta}_{\mu} \Lambda_{0}^{\mu \nu} \Delta_{\nu}
$$

where円

$$
\begin{aligned}
\Lambda_{0}^{\mu \nu}= & -\left(i \not \partial-M_{\Delta}\right) g^{\mu \nu}-i A\left(\gamma^{\mu} \partial^{\nu}+\gamma^{\nu} \partial^{\mu}\right) \\
& -\frac{1}{2} i\left(3 A^{2}+2 A+1\right) \gamma^{\mu} \not \partial \gamma^{\nu}-\left(3 A^{2}+3 A+1\right) M_{\Delta} \gamma^{\mu} \gamma^{\nu},
\end{aligned}
$$

with $A\left(\neq-\frac{1}{2}\right)$ a parameter. Note the difference in an overall minus sign from some recent work [4. 15], as already noticed in Ref. [8]. This ensures that the physical spatial components of the $\Delta$ field behave correctly like a Dirac field and the resulting Hamiltonian is positive definite. The Lagrangian is invariant under the so-called point transformation [2],

$$
\begin{aligned}
\Delta_{\mu} & \rightarrow \Delta_{\mu}^{\prime}=\Delta_{\mu}+a \gamma_{\mu} \gamma_{\nu} \Delta^{\nu}, \\
A & \rightarrow A^{\prime}=\frac{A-2 a}{1+4 a}
\end{aligned}
$$

\footnotetext{
${ }^{1}$ We use the conventions of Bjorken and Drell [14].
} 
where $a$ is arbitrary except $a \neq-1 / 4$. Note that the above transformation is not a symmetry of the Lagrangian since it changes the parameter $A$. Thus, it is more precise to call it a field redefinition which does not change physical quantities and, as a result, the parameter $A$ has no physical consequences.

The low-energy Lagrangian for the $\pi N \Delta$ system can be written as an expansion in derivatives of the pion field. Chiral symmetry $(S U(2) \otimes S U(2)$ realized in the non-linear form), Lorentz invariance and parity dictate the form of the Lagrangian which can be written as the sum of two parts, one $\left(\mathcal{L}_{\pi N}\right)$ involves only pions and nucleons and the other $\left(\mathcal{L}_{\Delta}\right)$ also contains $\Delta$ isobars. That is

$$
\mathcal{L}=\mathcal{L}_{\pi N}+\mathcal{L}_{\Delta}
$$

Up to two derivatives on the pion field, we have

$$
\begin{aligned}
\mathcal{L}_{\pi N}= & \bar{N}\left(i \mathcal{D}+g_{\mathrm{A}} \gamma^{\mu} \gamma_{5} a_{\mu}-M\right) N+\frac{f_{\pi}^{2}}{4} \operatorname{tr}\left(\partial_{\mu} U^{\dagger} \partial^{\mu} U\right) \\
& +\frac{1}{4} m_{\pi}^{2} f_{\pi}^{2} \operatorname{tr}\left(U+U^{\dagger}-2\right)+\frac{1}{M} \bar{N}\left(\beta_{\pi} \operatorname{tr}\left(\partial_{\mu} U^{\dagger} \partial^{\mu} U\right)-\kappa_{\pi} v_{\mu \nu} \sigma^{\mu \nu}\right) N \\
& +\frac{\kappa_{1}}{2 M^{2}}\left[i \bar{N} \gamma_{\mu} \mathcal{D}_{\nu} N \operatorname{tr}\left(a^{\mu} a^{\nu}\right)+\text { h.c. }\right]+\frac{\kappa_{2}}{M} m_{\pi}^{2} \bar{N} N \operatorname{tr}\left(U+U^{\dagger}-2\right),
\end{aligned}
$$

where the nucleon covariant derivative is $\mathcal{D}_{\mu} N=\partial_{\mu} N+i v_{\mu} N$, the trace "tr" is over $S U(2)$ isospin indices, $f_{\pi} \approx 93 \mathrm{MeV}$ is the pion decay constant, $g_{\mathrm{A}} \approx 1.26$ is the axial coupling, and $\beta_{\pi}, \kappa_{\pi}, \kappa_{1}$ and $\kappa_{2}$ are additional higher-order couplings. Note that we have organized the terms in accordance with Georgi's naive dimensional analysis [16]. In addition to the chiral invariant terms, we have introduced symmetry-breaking terms proportional to the square of the pion mass $m_{\pi}^{2}$. The axial and vector fields are defined in terms of the pion field by

$$
\begin{aligned}
a_{\mu} & \equiv-\frac{i}{2}\left(\xi^{\dagger} \partial_{\mu} \xi-\xi \partial_{\mu} \xi^{\dagger}\right)=a_{\mu}^{\dagger}=\frac{1}{2} \boldsymbol{a}_{\mu} \cdot \boldsymbol{\tau}=\frac{1}{f_{\pi}} \partial_{\mu} \pi+\cdots, \\
v_{\mu} & \equiv-\frac{i}{2}\left(\xi^{\dagger} \partial_{\mu} \xi+\xi \partial_{\mu} \xi^{\dagger}\right)=v_{\mu}^{\dagger}=\frac{1}{2} \boldsymbol{v}_{\mu} \cdot \boldsymbol{\tau}=-\frac{i}{2 f_{\pi}^{2}}\left[\pi, \partial_{\mu} \pi\right]+\cdots \\
v_{\mu \nu} & =-i\left[a_{\mu}, a_{\nu}\right]=\partial_{\mu} v_{\nu}-\partial_{\nu} v_{\mu}+i\left[v_{\mu}, v_{\nu}\right]
\end{aligned}
$$

with $\pi \equiv \frac{1}{2} \boldsymbol{\pi} \cdot \boldsymbol{\tau}$ and $U(x)=\xi \xi=\exp \left(2 i \pi(x) / f_{\pi}\right)$. 
The part of the chiral Lagrangian involving the $\Delta$ is

$$
\mathcal{L}_{\Delta}=\bar{\Delta}_{\mu}^{a} \Lambda_{a b}^{\mu \nu} \Delta_{\nu}^{b}+h_{\mathrm{A}}\left(\overline{\boldsymbol{\Delta}}_{\mu} \cdot \boldsymbol{a}_{\nu} \Theta^{\mu \nu} N+\bar{N} \Theta^{\mu \nu} \boldsymbol{a}_{\mu} \cdot \boldsymbol{\Delta}_{\nu}\right)+\tilde{h}_{\mathrm{A}} \bar{\Delta}_{\mu}^{a} \Gamma^{\mu \nu \sigma} \gamma_{5} a_{\sigma} \Delta_{\nu}^{a}
$$

where $h_{\mathrm{A}}$ and $\tilde{h}_{\mathrm{A}}$ are the $\pi N \Delta$ and $\pi \Delta \Delta$ couplings. We have found it convenient to define the $\Delta$ in terms of three two-component spinors in isospin space (see the Appendix) by setting $\boldsymbol{\Delta}_{\mu}=\boldsymbol{T} \Delta_{\mu}$ using the standard $2 \times 4$ isospin $\frac{3}{2}$ to $\frac{1}{2}$ transition matrix:

$$
\left\langle\frac{1}{2} t|\boldsymbol{T}| \frac{3}{2} t_{\Delta}\right\rangle \equiv \sum_{\lambda}\left\langle 1 \lambda \frac{1}{2} t \mid \frac{3}{2} t_{\Delta}\right\rangle \boldsymbol{e}_{\lambda}
$$

with the isospin spherical unit vectors $\boldsymbol{e}_{0}=\boldsymbol{e}_{z}$ and $\boldsymbol{e}_{ \pm 1}=\mp\left(\boldsymbol{e}_{x} \pm i \boldsymbol{e}_{y}\right) / \sqrt{2}$. The kernel in the first term of (10), $\Lambda_{a b}^{\mu \nu}$, is obtained from $\Lambda_{0}^{\mu \nu} \delta_{a b}$ of (3) by replacing the ordinary derivative with the covariant derivative

$$
\mathcal{D}_{\mu} \boldsymbol{\Delta}_{\nu}=\partial_{\mu} \boldsymbol{\Delta}_{\nu}+i v_{\mu} \boldsymbol{\Delta}_{\nu}-\boldsymbol{v}_{\mu} \times \boldsymbol{\Delta}_{\nu}
$$

We note in passing that

$$
\boldsymbol{T}^{\dagger} \cdot \mathcal{D}_{\mu} \boldsymbol{\Delta}_{\nu}=\partial_{\mu} \Delta_{\nu}+i \boldsymbol{v}_{\mu} \cdot \boldsymbol{t}^{\left(\frac{3}{2}\right)} \Delta_{\nu}
$$

where $\boldsymbol{t}^{\left(\frac{3}{2}\right)}$ are the generators of the isospin- $\frac{3}{2}$ algebra. For the $\pi N \Delta$ term we employ the standard definition 20

$$
\Theta_{\mu \nu}=g_{\mu \nu}+\frac{1}{2}[(4 Z+1) A+2 Z] \gamma_{\mu} \gamma_{\nu}
$$

which defines the off-shell $Z$ parameter. For the $\pi \Delta \Delta$ interaction we have defined

$$
\begin{aligned}
\Gamma^{\mu \nu \sigma} \equiv & g^{\mu \nu} \gamma^{\sigma}+\left[\left(4 Z_{2}+1\right) A+2 Z_{2}\right]\left(\gamma^{\mu} g^{\nu \sigma}+\gamma^{\nu} g^{\mu \sigma}\right) \\
& -\left[\left(2 Z_{2}+\frac{1}{2}\right) A+Z_{2}+\left(4 Z_{3}+\frac{1}{2}\right)\left(A^{2}+A\right)+Z_{3}\right] \gamma^{\mu} \gamma^{\sigma} \gamma^{\nu}
\end{aligned}
$$

which involves two additional off-shell parameters $Z_{2}$ and $Z_{3}$. Each of the three terms in $\mathcal{L}_{\Delta}$ is invariant under the field redefinition (4), indeed the $Z$ parameters have been introduced precisely to preserve this invariance. To make this Letter self-contained, the chiral invariance of the Lagrangian warrants a brief discussion; this is deferred to the Appendix. 
In what follows we show that, besides the $A$ parameter, the dependence on the $Z$ parameters can also be absorbed in the other parameters in the Lagrangian. To achieve this, we integrate out the $\Delta$ field and examine the resulting equivalent $\pi N$ Lagrangian. This can be done by performing the standard Gaussian integration since the Lagrangian (10) is quadratic in the $\Delta$ field to the order we keep. Because the off-shell vertices connected to on-shell external lines vanish, it is sufficient to discuss the case without an external source for the $\Delta$.

First, in the standard functional integral, we may redefine the $\Delta$ field as in (田) with $a=-(A+1) / 2$ so that $A^{\prime}=-1$. Since the Jacobian is constant we see explicitly that the dependence on the parameter $A$ is removed. Now we may formally identify

$$
\eta_{\mu}(x)=h_{\mathrm{A}} \Theta_{\mu \nu} \boldsymbol{T}^{\dagger} \cdot \boldsymbol{a}^{\nu} N
$$

as a "source" for the $\Delta$ field. Performing the $\Delta$ functional integration then yields an equivalent Lagrangian

$$
\begin{aligned}
\int d^{4} x \mathcal{L}_{\equiv}= & \int d^{4} x \mathcal{L}_{\pi N}-i \operatorname{Tr} \ln \left(K \Lambda_{0}^{-1}\right) \\
& -\int d^{4} x d^{4} y \bar{\eta}^{\mu}(x) G_{\mu \nu}(x, y) \eta^{\nu}(y)
\end{aligned}
$$

where, in the second term, the trace "Tr" is over spacetime, isospin, and vector-spinor indices and it has been normalized to vanish when the pion field is set to zero. The inverse of the free propagator is given by $4,15,17$

$$
\left(\Lambda_{0}^{-1}\right)_{\mu \nu}(x, y)=S_{\mu \nu}(x) \delta^{4}(x-y)
$$

with

$$
S_{\mu \nu}(x)=\frac{1}{i \not \partial-M_{\Delta}+i \epsilon}\left[-g_{\mu \nu}+\frac{1}{3} \gamma_{\mu} \gamma_{\nu}+\frac{i}{3 M_{\Delta}}\left(\gamma_{\mu} \partial_{\nu}-\gamma_{\nu} \partial_{\mu}\right)-\frac{2}{3 M_{\Delta}^{2}} \partial_{\mu} \partial_{\nu}\right] \text {, }
$$

and $G_{\mu \nu}(x, y)$ is the inverse of the kernal

$$
K^{\mu \nu}(x, y) \equiv T_{a}^{\dagger}\left(\Lambda_{a b}^{\mu \nu}+\tilde{h}_{\mathrm{A}} \Gamma^{\mu \nu \sigma} \gamma_{5} a_{\sigma} \delta_{a b}\right) T_{b} \delta^{4}(x-y)
$$


The trace term in (17) corresponds to short-distance physics since the mass of the $\Delta$ sets the relevant scale. This trace can be expanded in derivatives of the pion field, resulting in local terms that can be absorbed into the coefficients in $\mathcal{L}_{\pi N}$ as in Ref. [18. Up to two derivatives on the pion field, one can replace $G_{\mu \nu}(x, y)$ in the last term of (17) with the free propagator given in (19). This is the case since the terms involving the pion field in the denominator of $G_{\mu \nu}(x, y)$ can be treated perturbatively as a result of the fact that a derivative on the pion field is assciated with a factor of $1 /\left(4 \pi f_{\pi}\right)$ from the loop integral [19]. The corresponding contribution to the Lagrangian $\mathcal{L}_{\equiv}$ can then be written as

$$
\mathcal{L}_{1}(x)=-h_{\mathrm{A}}^{2} \bar{N} \boldsymbol{T} \cdot \boldsymbol{a}_{\mu} \Theta^{\mu \lambda} S_{\lambda \sigma} \Theta^{\sigma \nu} \boldsymbol{T}^{\dagger} \cdot \boldsymbol{a}_{\nu} N
$$

Noticing that

$$
\gamma_{\mu} S^{\mu \nu}=S^{\nu \mu} \gamma_{\mu}=\frac{1}{3 M_{\Delta}}\left(\frac{2}{M_{\Delta}} i \partial^{\nu}-\gamma^{\nu}\right)
$$

one finds

$$
\begin{aligned}
\Theta_{\mu \lambda} S^{\lambda \sigma} \Theta_{\sigma \nu}=S_{\mu \nu} & +\frac{2}{3 M_{\Delta}^{2}}\left(Z+\frac{1}{2}\right)\left[M_{\Delta} \gamma_{\mu} \gamma_{\nu}-\left(\gamma_{\mu} i \partial_{\nu}+\gamma_{\nu} i \partial_{\mu}\right)\right] \\
& -\frac{2}{3 M_{\Delta}^{2}}\left(Z+\frac{1}{2}\right)^{2} \gamma_{\mu}\left(2 M_{\Delta}-i \not \partial\right) \gamma_{\nu}
\end{aligned}
$$

Putting (23) into (21) and using the nucleon equation of motion to remove derivatives on the nucleon field, we find

$$
\begin{aligned}
\mathcal{L}_{1}(x)= & -h_{\mathrm{A}}^{2} \bar{N} \boldsymbol{T} \cdot \boldsymbol{a}_{\mu} S^{\mu \nu} \boldsymbol{T}^{\dagger} \cdot \boldsymbol{a}_{\nu} N-\frac{8}{9} \frac{h_{\mathrm{A}}^{2}}{M_{\Delta}^{2}}\left(Z^{2}-\frac{1}{4}\right)\left[i \bar{N} \gamma_{\mu} \partial_{\nu} N \operatorname{tr}\left(a^{\mu} a^{\nu}\right)+h . c .\right] \\
& +C\left[\bar{N} N \operatorname{tr}\left(\partial_{\mu} U^{\dagger} \partial^{\mu} U\right)-2 \bar{N} v_{\mu \nu} \sigma^{\mu \nu} N\right]
\end{aligned}
$$

where the constant

$$
C \equiv \frac{2}{9} \frac{h_{\mathrm{A}}^{2}}{M_{\Delta}^{2}}\left[\left(Z+\frac{1}{2}\right)^{2}\left(2 M_{\Delta}+M\right)-\left(Z+\frac{1}{2}\right) M_{\Delta}\right]
$$

The second and third terms on the right of (24) can clearly be absorbed into the corresponding terms in $\mathcal{L}_{\pi N}$ of (6). For example, we can define $\beta_{\pi}=\beta_{\pi}^{\prime}-M C$ so that only $\beta_{\pi}^{\prime}$ is 
physically significant. Thus, one arrives at a nonlocal Lagrangian for the $\pi N$ system given by

$$
\mathcal{L}_{\equiv}(x)=\mathcal{L}_{\pi N}(x)-h_{\mathrm{A}}^{2} \bar{N} \boldsymbol{T} \cdot \boldsymbol{a}_{\mu} S^{\mu \nu} \boldsymbol{T}^{\dagger} \cdot \boldsymbol{a}_{\nu} N
$$

Since this $Z$-independent Lagrangian is equivalent to that in (5), we conclude that the $Z$ parameter is physically irrelevant.

We note, however, that it is not convenient to work with a nonlocal Lagrangian as in (26) since it is hard to maintain the various symmetries and to organize the Lagrangian according to the naive dimensional analysis [16]. Although one may still expand the second term in (26) in a Taylor series in derivatives of the pion field, each derivative will be suppressed by $1 /\left(M_{\Delta}-M\right)$ rather than $1 / M$. This cannot be a useful expansion since it violates the naive dimensional analysis [16 and any truncation would result in large errors. This shows that one needs to treat the $\Delta$ as a dynamical degree of freedom in agreement with Refs. [8,10,11]. Thus our analysis indicates that the original effective Lagrangian (5) should be used including the $\Delta$ field explicitly and making some convenient choice for the parameters $A$ and $Z$ (such as $A=-1, Z=-1 / 2)$ since they are not relevant to the physics.

For clarity we have limited our discussions to terms with two derivatives on the pion field, our results can be generalized nevertheless. Consider the $\pi N \Delta$ or $\pi \Delta \Delta$ vertex in (10). If it is attached to a $\Delta$ line in a Feynman diagram of an arbitrary order, the pole of the $\Delta$ propagator will be removed according to $(22)$ if the vertex contains a $\gamma_{\mu}$ that is contracted with the propagator. This is the case for the $Z, Z_{2}$, and $Z_{3}$ terms where the $\Delta$ line will shrink to a point and generate local vertices which can be combined with similar vertices in the Lagrangian. Thus the $Z, Z_{2}$, and $Z_{3}$ parameters can all be subsumed in the infinite number of parameters in the Lagrangian to all orders.

To summarize, we have demonstrated that the $\Delta$ should be treated as a dynamical degree of freedom and all the off-shell parameters $Z, Z_{2}$, and $Z_{3}$ are redundant since they can be absorbed into other parameters in the Lagrangian. Our results should pave the way for systematic inclusion of $1 / M$ corrections involving the $\Delta$ isobar in heavy-baryon ChPT and 
in calculations for nuclear matter and finite nuclei where the $1 / M$ corrections are crucial.

We thank D.K. Griegel, S. Jeon, J.I. Kapusta and B.D. Serot for useful comments and stimulating discussions. We acknowledge support from the Department of Energy under grant No. DE-FG02-87ER40328.

\section{APPENDIX:}

To be self-contained, we briefly discuss the transformation of the various fields under a non-linear realization [20] of $S U(2)_{\mathrm{L}} \otimes S U(2)_{\mathrm{R}}$ symmetry. More details may be found in e.g. Ref. 21]. With $L \in S U(2)_{\mathrm{L}}$ and $R \in S U(2)_{\mathrm{R}}$, one has the mapping

$$
L \otimes R: \quad\left(\xi, N, \Delta_{\mu}\right) \longrightarrow\left(\xi^{\prime}, N^{\prime}, \Delta_{\mu}^{\prime}\right)
$$

where

$$
\begin{aligned}
\xi^{\prime}(x) & =L \xi(x) h^{\dagger}(x)=h(x) \xi(x) R^{\dagger}, \\
N^{\prime}(x) & =h(x) N(x) \\
\boldsymbol{\Delta}_{\mu}^{\prime}(x) & =\frac{1}{2} h \operatorname{tr}\left(h \boldsymbol{\tau} h^{\dagger} \boldsymbol{\tau}\right) \cdot \boldsymbol{\Delta}_{\mu}(x) .
\end{aligned}
$$

The second equality in (A2) defines $h(x)$ as a function of $L, R$, and the local pion field: $h(x)=h(\boldsymbol{\pi}(x), L, R)$. The pseudoscalar nature of the pion field implies then $h(x) \in S U(2)_{\mathrm{V}}$, with $S U(2)_{\mathrm{V}}$ the unbroken vector subgroup of $S U(2)_{\mathrm{L}} \otimes S U(2)_{\mathrm{R}}$. Eqs. (A3) and (A4) ensure that the nucleon transforms linearly under $S U(2)_{\mathrm{V}}$ as an isospin Pauli-spinor and the $\Delta$ as an isospin vector Pauli-spinor. (Note that $\frac{1}{2} \operatorname{tr}\left(h \boldsymbol{\tau} h^{\dagger} \boldsymbol{\tau}\right)$ provides the orthogonal transformation of the isovector part of the field). Writing out the $\boldsymbol{\Delta}$ field explicitly, one finds

$$
\begin{aligned}
& \Delta_{\mu}^{1}+i \Delta_{\mu}^{2}=\frac{1}{\sqrt{3}}\left(\begin{array}{c}
\sqrt{2} \Delta_{\mu}^{0} \\
\sqrt{6} \Delta_{\mu}^{-}
\end{array}\right) \\
& \Delta_{\mu}^{1}-i \Delta_{\mu}^{2}=-\frac{1}{\sqrt{3}}\left(\begin{array}{c}
\sqrt{6} \Delta_{\mu}^{++} \\
\sqrt{2} \Delta_{\mu}^{+}
\end{array}\right)
\end{aligned}
$$




$$
\Delta_{\mu}^{3}=\sqrt{\frac{2}{3}}\left(\begin{array}{c}
\Delta_{\mu}^{+} \\
\Delta_{\mu}^{0}
\end{array}\right) .
$$

It is useful to note the following properties of the isospin transition matrix defined in (11):

$$
T_{a}^{\dagger} T_{a}=\mathbf{1}, \quad T_{a} T_{b}^{\dagger}=\delta_{a b}-\frac{1}{3} \tau_{a} \tau_{b},
$$

where 1 represents the $4 \times 4$ unit matrix. Also the transformation properties of the axial vector and vector fields are $a_{\mu}^{\prime}=h a_{\mu} h^{\dagger}$ and $v_{\mu}^{\prime}=h v_{\mu} h^{\dagger}-i h \partial_{\mu} h^{\dagger}$. 


\section{REFERENCES}

[1] G. Höhler, Pion-Nucleon Scattering, Landolt-Börnstein, New Series, ed. H. Schopper, Vol. I/9 b2 (Springer-Verlag, Berlin, 1983).

[2] L. M. Nath, B. Etemadi, and J. D. Kimel, Phys. Rev. D3 (1970) 2153.

[3] M. G. Olsson and E. T. Osypowski, Nucl. Phys. B101 (1975) 136.

[4] M. Benmerrouche, R. M. Davidson, and N. C. Mukhopadhyay, Phys. Rev. C39 (1989) 2339.

[5] P. F. A. Goudsmit, H. J. Leisi, E. Matsinos, B. L. Birbrair, and A. B. Gridnev, Nucl. Phys. A575 (1994) 673.

[6] See, e.g., G. P. Lepage, "What is renormalization?", in From Actions to Answers, edited by T. DeGrand and D. Toussaint (World Scientific, 1989); H. Georgi, Ann. Rev. Nuc. Part. Sci. 43, 209 (1993); S. Weinberg, The Quantum Theory of Fields, vol. I: Foundations (Cambridge University Press, New York, 1995).

[7] S. Weinberg, Physica A96 (1979) 327; J. Gasser and H. Leutwyler, Ann. Phys. 158 (1984) 142; Nucl. Phys. B250 (1985) 465, 517, 539.

[8] M. K. Banerjee and J. Milana, Phys. Rev. D52 (1995) 6451.

[9] E. Jenkins and A. V. Manohar, Phys. Lett. B255 (1991) 558.

[10] E. Jenkins and A. V. Manohar, Phys. Lett. B259 (1991) 353; E. Jenkins, Nucl. Phys. B368 (1992) 190.

[11] C. Ordonez, L. Ray, and U. van Kolck, Phys. Rev. Lett. 72 (1994) 1982.

[12] V. Bernard, N. Kaiser, and U. Meissner, Phys. Lett. B378 (1996) 337; B382 (1996) 19.

[13] P. A. Moldauer and K. M. Case, Phys. Rev. 102 (1956) 279.

[14] J. D. Bjorken and S. D. Drell, Relativistic Quantum Fields (McGraw-Hill, New York, 
1965).

[15] D. K. Griegel, Phys. Rev. C43 (1990) 904.

[16] H. Georgi, Phys. Lett. B298 (1993) 187.

[17] H. Pilkuhn, The Interactions of Hadrons (North Holland, Amsterdam, 1967).

[18] R. J. Furnstahl, H.-B. Tang, and B. D. Serot, Phys. Rev. C52 (1995) 1368.

[19] A. V. Manohar and H. Georgi, Nucl. Phys. B234 (1984) 189.

[20] S. Coleman, J. Wess, and B. Zumino, Phys. Rev. 177 (1969) 2239; C. Callan, S. Coleman, J. Wess, and B. Zumino, Phys. Rev. 177 (1969) 2247.

[21] R. J. Furnstahl, B. D. Serot, and H.-B. Tang, uucl-th/9608035. 